Rapid Reviews COVID-19

\title{
Review 1: "The Personal Responsibility Pandemic: Centering Solidarity in Public Health and Employment Law"
}

Pamela Egan ${ }^{1}$, Ken Jacobs ${ }^{1}$

${ }^{1}$ University of California, Berkeley, Center for Labor Research and Education

Published on: Sep 14, 2020

DOI: $10.1162 / 2 \mathrm{e} 3983 f 5.6 \mathrm{c} 242 \mathrm{abe}$

License: Creative Commons Attribution 4.0 International License (CC-BY 4.0). 


\section{$\underline{\text { RR:C19 Evidence Scale rating by reviewer: }}$}

- Strong. The main study claims are very well-justified by the data and analytic methods used. There is little room for doubt that the study produced has very similar results and conclusions as compared with the hypothetical ideal study. The study's main claims should be considered conclusive and actionable without reservation.

$* * * * * * * * * * * * * * * * * * * * * * * * * * * * * * * * * * * * * * *$

\section{Review:}

In “The Personal Responsibility Pandemic," Professors Lindsay F. Wiley and Samuel R. Bagenstos make a strong case for adopting a solidarity-based reframing of public health interventions, employment law and anti-discrimination law in response to the COVID-19 pandemic.

The authors draw a clear nexus between public health, employment, and job-based discrimination. Their exposition of the dominant personal responsibility and fault/responsibility paradigms undergirding the current public health system and employment and discrimination law makes a valuable contribution to our understanding of those systems' failure to effectively address the pandemic, especially for Black, Latinix, Native American, immigrant, low-wage/low-income, female, differently-abled, older, and justice- system involved communities and workers. Their analysis clarifies the mechanisms by which the personal responsibility approach fragments communities and is not only inadequate to address public health challenges, inequality, and economic wellbeing, but exacerbates both health and economic disparities for vulnerable and disadvantaged groups.

Wiley and Bagenstos' alternative, solidarity-based approach to public health interventions, workplace protections, and support structures rooted in an acknowledgement of "interdependence among individuals and groups," "reciprocity of support," and "connectedness between individuals and their communities" is detailed and well-reasoned. The authors demonstrate that public health threats and effective interventions share a communal nature, and their argument for widely and equitably shared responsibility for burdens and benefits offers a useful framework for both policy and program development in public health and employment and discrimination law reform. 
The authors' set of initial proposals to protect workers' health and safety, socialize the costs of protecting essential workers and those who cannot work during the pandemic, prevent retaliation against workers, and fairly distribute costs and benefits across categories of workers are specific, clear, and actionable by policy makers and public health actors.

We consider "The Personal Responsibility Pandemic" strong in both analysis and conclusions and we recommend publication.

We do note the absence of policy solutions that support collective action. This gap stands out in an article focused on a solidarity-based approach to public health, and one that clearly articulates the role of worker fear in deterring reports of abuse. The article would benefit from a discussion of solutions at the intersection of collective action, employment and discrimination law, and public health, such as those found in Sharon Block and Benjamin Sachs' report "Worker Power and Voice in the Pandemic Response" (Labor and Worklife Program, Harvard Law School, 2020), and recent research findings that the presence of a union correlated with 30\% fewer COVID-19 deaths, increased availability of PPE, and $42 \%$ lower infection rates in nursing homes in New York State (Dean, Adam, et al. "Mortality Rates From COVID-19 Are Lower In Unionized Nursing Homes." Health Affairs, 2020, doi:10.1377/hlthaff.2020.01011). 